\title{
Design and construction Optically Gas Laser
}

\author{
Nahla.A. Al Jabar* \\ Kamal.H.Latif * \\ Bushra R.Mhdi* \\ Huda A. Al Jabar** \\ Basher R.Mhdi*
}

Received 2, June, 2011

Accepted 26, June, 2012

\begin{abstract}
:
In this work the design and construction of a flash photolysis pulsed $\mathrm{HCl}$ laser was presented. The parameters of the pumping source and discharge current density was obtained, which sufficient to shift the flash lamp spectrum towards uv portion of spectrum. The maximum pulse laser energy parameters was measured.

Total pressure and ratio of active gases to optimized the output pulse energy were measured, where at 125 mbar of total pressure and 1:7:14 $\mathrm{Cl}_{2}: \mathrm{H}_{2}: \mathrm{He}$ ratio, the laser energy was measured to be $200 \mathrm{~mJ}$ at pumping four flash lamps energy in the order of $6400 \mathrm{~J}$.

The resonator consists of copper a near hemispherical mirror with the radius of curvature $3 \mathrm{~m}$ coated by gold and reflectivity $98 \%$, the output coupler sapphire mirror of $63 \%$ reflectivity . Total efficiency of the system was measured to be $0.0125 \%$.
\end{abstract}

\section{Key words: Pulse gas laser, Hcl laser.}

\section{Introduction:}

The chemical laser proved to be one of the most intensive laser systems during the last five decades. This is due to many facts, namely; being among the most powerful, high efficiency lasers, reaching $60 \mathrm{KJ}$ in pulse and 1 $\mathrm{KW}$ in $\mathrm{CW}$ modes [1,2].

The high energy of chemical laser draw attention of many researchers to develop systems for many fields of applications particularly for industrial and military uses. The wavelength of such kind of lasers fall in the range $(2-5) \mu \mathrm{m}$ which makes them of immense importance in the far field laser target treatment, this is due to the limited attenuation for this range of wavelength in space is very low [3]. Chemical compounds are able to store large amount of energy which may be partially released in exothermic chemical reactions [4].

A chemical laser is a laser in which population inversion is directly produced by an elementary chemical

reaction. Accordingly, a chemical laser is capable of yielding very-high power densities with significantly small reaction initiation energies in comparison to the emitted laser energy [5]. They operate on a rotationalvibrational transition in molecule. This is because many exothermic gas-phase reactions liberate their energy through a stretching vibration in the newly formed bond [6].

In chemical laser, the initiation energy only serves to prepare chemical species that react exothermically to produce the lasing active medium. Initiation efficiency of chemical lasers being as high as $\geq 200 \%$, whereas for solid state and $\mathrm{CO}_{2}$ gas lasers it is $\leq$ $5 \%$ and $25 \%$ respectively $[7,8]$.

The first chemical laser was demonstrated by V.V.Kasper et.al. in 1965[9]. It has used the flash lamp to initiate $\mathrm{HCl}$ laser using chain reaction of $\left(\mathrm{Cl}_{2}-\mathrm{H}_{2}\right)$ mixture. The flash lamp

* Ministry of Science and Technology.

** University of Baghdad/College of Science for Women. 
delivered an input energy of about (3800J) which was found to yield a laser pulse of 20J energy.. In the 1968 the same previous research team utilized the chain reaction of $\left(\mathrm{Cl}_{2}-\mathrm{H}_{2}\right)$ mixture. The flash lamp and laser tube both were made of quartz. They were placed on the two axes of an elliptical aluminum reflector. the input energy of the lamp was about $1100 \mathrm{~J}$ to give about 12J laser output pulse [10].

During the eighties, the technique of using lasers to initiate the reaction instead of flash lamps appears in the work of. Hirokozn, 1984. The chain reaction, which is initiated by UV flash using $\mathrm{F}_{2}-\mathrm{H}_{2}$ mixture, to achieve (14.6) J/Pluse laser output energy at $(0.7 \mathrm{~atm})$ [6].In 1986 A.S.Bashkin, utilized the chain reaction for $\left(\mathrm{D}_{2}-\mathrm{F}_{2}\right)$ mixture, using flash lamp.In 1996 B.G.Bravy, utilized the chain reaction for $\mathrm{F}_{2}-\mathrm{H}_{2}$ mixture, initiated by flash lamp to get $1 \mathrm{KJ}$ output laser energy at total pressure of 0.7 atm, with 50 litter. The beam divergence was $10^{-4} \mathrm{rad}$ [10].A ClF and $\mathrm{H}_{2}$ mixture chain reaction, initiated by using $\mathrm{XeCl}$ laser (308 nm), $50 \mathrm{~J} / \ell$ laser energy pulse, was utilized by E.B. Gorden in 1997 [11].

\section{Materials and Methods}

The first chemical laser has been made on the basis of a reaction proceeding in the mixture of a molecular hydrogen and a chlorine $\mathrm{H}_{2}+\mathrm{Cl}_{2}$. The aforementioned, process is a classical example of a chain chemical process. It proceeds in the following manner: The chlorine molecules are decomposed into atoms under the action of uv radiation. These atoms will be responsible for initiating the reaction with a long chain as follows[12]:

$$
\mathrm{Cl}_{2}+\mathrm{hv} \rightarrow 2 \mathrm{Cl}
$$

$\mathrm{Cl}+\mathrm{H}_{2} \rightarrow \mathrm{HCl}+\mathrm{H}$

cold reaction (endothermic reaction)

$-\Delta \mathrm{H}=1 \mathrm{~K} \mathrm{cal} / \mathrm{mol} \quad \mathrm{E}_{\mathrm{A}}=5.5 \mathrm{~K}$ $\mathrm{cal} / \mathrm{mol}$

$\mathrm{H}+\mathrm{Cl}_{2} \rightarrow \mathrm{HCl}^{*}+\mathrm{Cl}$

hot reaction (exothermic reaction)

$$
\begin{aligned}
& -\Delta \mathrm{H}=45.1 \mathrm{~K} \mathrm{cal} / \mathrm{mol} \quad \mathrm{E}_{\mathrm{A}}= \\
& 1.8 \mathrm{~K} \mathrm{cal} / \mathrm{mol} \\
& \mathrm{HCl}+\mathrm{nhv} \rightarrow \mathrm{HCl}+(\mathrm{n}+1) \mathrm{hv}
\end{aligned}
$$

The $\mathrm{HCl}$ molecules are produced non excited in the first link of the process since no energy is being released here. However, a quite large amount of energy is released approximately $2 \mathrm{eV}$ in each elementary act in the second link, therefore, the $\mathrm{HCl}$ molecules are excited and consequently, the coherent emission is produced. Firstly, the chain process was realized just in the first chemical laser. However, as it turned out, the energy expenses for the production of chloride atoms was thousand times larger than the energy of the generated coherent emission.

The first link takes place slowly in that reaction. Accordingly, the rate of the chain is slower than the rate of processes which are responsible for deactivating the molecule excitation. The chemical reaction in the hydrogen chloride mixture is non-chain from the laser point of view.

This mechanism has three important effects on the operation of the laser, first of all: the rate of the pumping reaction ( 3 ) is limited by that of the slower preceding reaction (eq. 2) because of its activation energy of 5.5 $\mathrm{Kcal} / \mathrm{mol}$ [12]. Second: the inversion due to vibration ally excited $\mathrm{HCl}$ produced in reaction (eq.3) is necessarily diluted by "cold" $\mathrm{HCl}$ produced concurrently in reaction (eq. 2) for which $\left(-\Delta H+E_{A}\right)$ is insufficient to excite $\mathrm{HCl} V=1$. Third: the cyclic chain provided by reactions eqs 2,3 
multiplies many-fold the number of pumping reaction steps 3 that can obtained for each produced chlorine atom. It is implied that only a small number of input photons in the form of initiating light might be sufficient to produce a far large number of output photons, which are excited [six vibration levels $\mathrm{HCl}(\mathrm{V}=0-5)]$.

\section{Techniques for Initiating Chemical Laser Reactions}

Some types of reaction initiation techniques, which are applied to the gases either before or after mixing, are listed below-

\section{A: Flash Photolysis Initiation:-}

This method has been used for initiation of reaction in pulsed $\mathrm{HF}$ and $\mathrm{HCl}$ chemical lasers. Ultraviolet light is needed for flash photolytic initiation. The absorption spectra of all $\left(\mathrm{H}_{2}, \mathrm{~F}_{2}\right.$, $\mathrm{Cl}_{2}$ ) has been measured. It indicates that the $\mathrm{F}_{2}, \mathrm{Cl}_{2}$ molecules can dissociate by the flash or not [5].

A small portion of the flash lamp light is produced in the useful wavelength region. It can be absorbed by the fluorine molecule, or actually absorbed reactant. Consequently, the losses in the flash lamp make the overall efficiencies low, $\left(\eta_{\text {lamp }}=13 \%\right)$. However, photolysis initiation will produce specially uniform reaction. The electrical efficiency is greater than $4 \%$ [12]. The laser pulse is shorter than the flash lamp duration, and the time delay between initiation of the flash lamp and the laser threshold is quite variable. The pulse of the flash lamp and the laser pulse are shown in Fig.(1) [4].

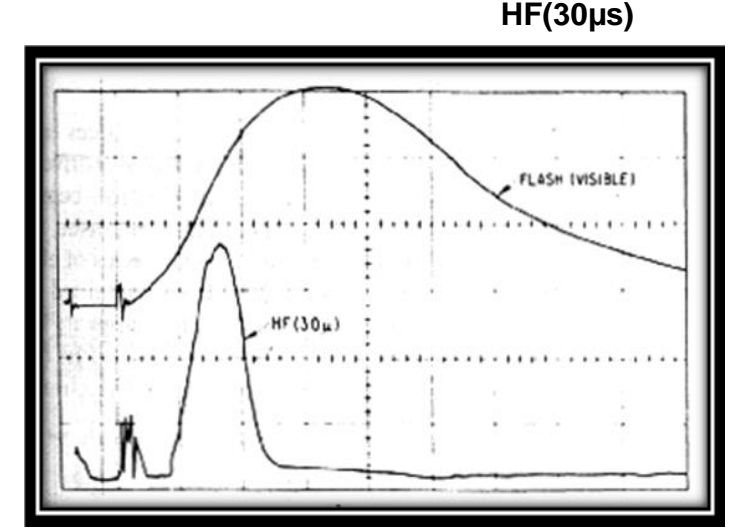

Fig. (1) the pulse of the flash lamp and the laser pulse as shown on the scope [4].

The flash photoplay is the simplest way of reaction for the active medium, although it is not the most efficient one. The pattern of the laser pulse shape is almost homogenous and the intensity is uniform. Therefore, it is suitable for far field target treatment, due to the limited beam divergence [4].

Increasing the number of flash lamps will increase the laser pulse energy. This is the most advantage characteristic of using flash lamp technique. It is especially true when the flash lamps are fixed outside the active medium (using Quartz tube). In addition, in such case, there is no interaction between the pumping source and the active medium. This will increase the pressure to higher range, than another pumping mechanism. The increasing of pressure will already increases the laser energy [10]. The electrical circuit, which can be used, is very simple and more reliable than other mechanism.

\section{B: Electrical - Discharge Initiation:-}

An electrical discharge provides an efficient means for initiating reaction in chemical laser which may be accomplished through either transverse or longitudinal discharge configuration [4]. 


\section{C: Electron - Beam Initiation :-}

HF chemical laser can lase with electrical laser efficiencies in excess of $100 \%$ by using a mixture of $\mathrm{H}_{2}$ and $\mathrm{F}_{2}$. $\mathrm{HF}$ laser initiated by using intense ebeams are considered of especial high efficiencies and can produce high output energy [13].

\section{D: Laser - Photolysis initiation :-}

Another technique for photolysis initiation is to irradiate the $\mathrm{H}_{2}-\mathrm{F}_{2}$ mixture with a frequency doubled ruby laser pulse at $347 \mathrm{~nm}$. The ruby-laser pulse which is directed longitudinally along the HF laser axis, photolysis as small percent of the $F_{2}$ to initiate the reaction [4].

\section{Experimental work and System Control}

The operation procedure of the laser system is electronically controlled through an electronic circuit. First of all, the control circuits triggered pneumatics value that is jointed to the chlorine bottle. The trigger time lasts for $100-250 \mathrm{msec}$. The variability of the trigger time is to control the chlorine pressure that pushes into the active medium.

Experimentally, when the chlorine bottle fuelled to about 1 bar and the active medium cavity evacuated to $10^{-2} \mathrm{mbar}$, then the chlorine pressure reaches (25-75) mbar. By these the operation time of the valve was varied from (100-250) msec respectively .Furthermore, it seems to be very reliable and simple to control. Secondly, after about $100 \mathrm{msec}$, the second valve of hydrogen-helium which is joint with the bottle of mixing, will open within a time varied from $0-300 \mathrm{msec}$. The He$\mathrm{H}_{2}$ is mixed primary using regulated flow meters to control the gas mixing ratio preciessly.

The third trigger was applied into thyristor switching circuit. It sends the pulse to a high voltage transformer within a time varied from $0.4-20 \mathrm{msec}$. The time of trigger lasts for nearly $80 \mu \mathrm{sec}$. The generated pulse was used to break down the gas resistance at four flash lamps so that charged capacitors will discharge through them.

The H.V discharge trigger circuit and the electronic controlling circuit are shown in Fig.(2).

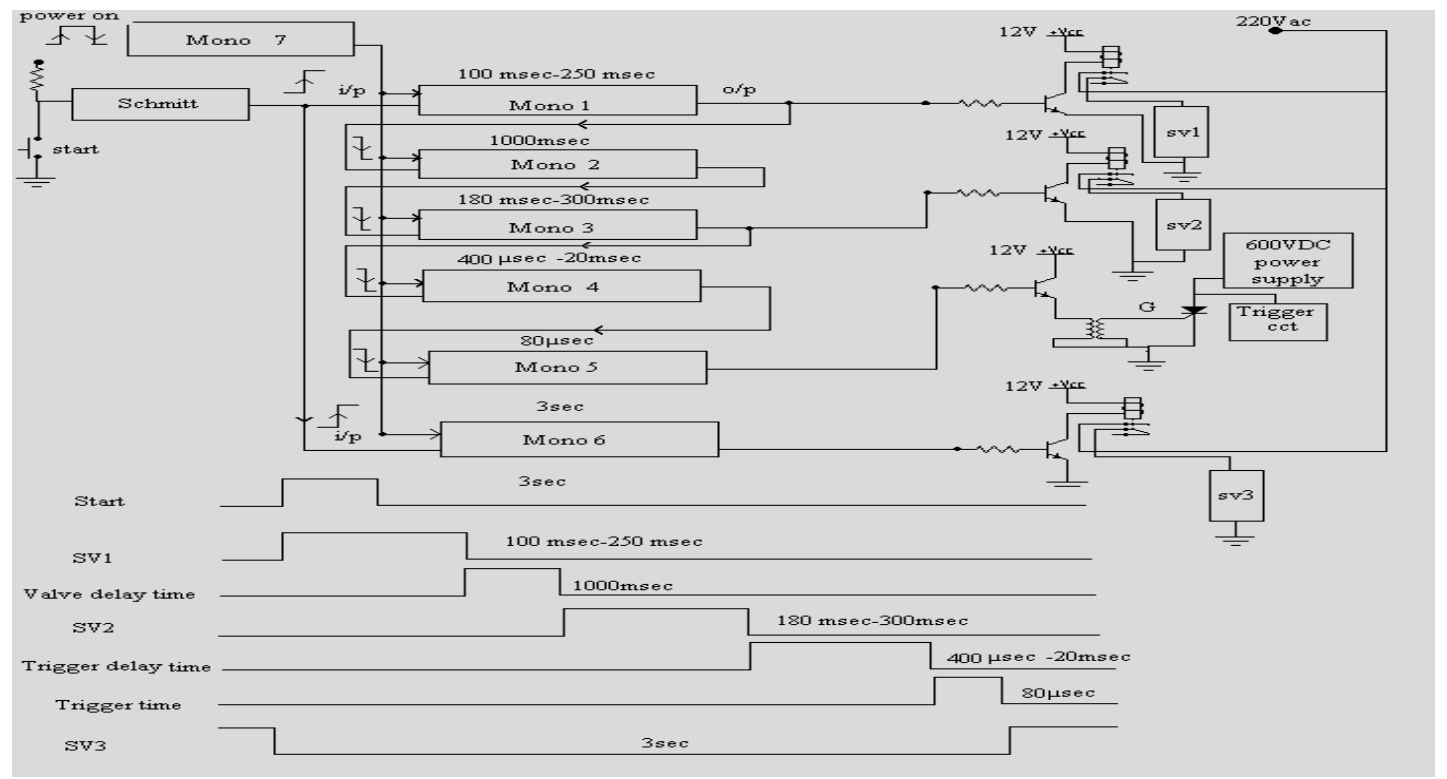

Fig. ( 2 ) The electronic equivalent circuit of the control system 


\section{Laser Cavity Design}

The active medium consists of a high optical quality quartz tube of $10 \mathrm{~cm}$ diameter and $100 \mathrm{~cm}$ length. The two ends were closed by two o-ring

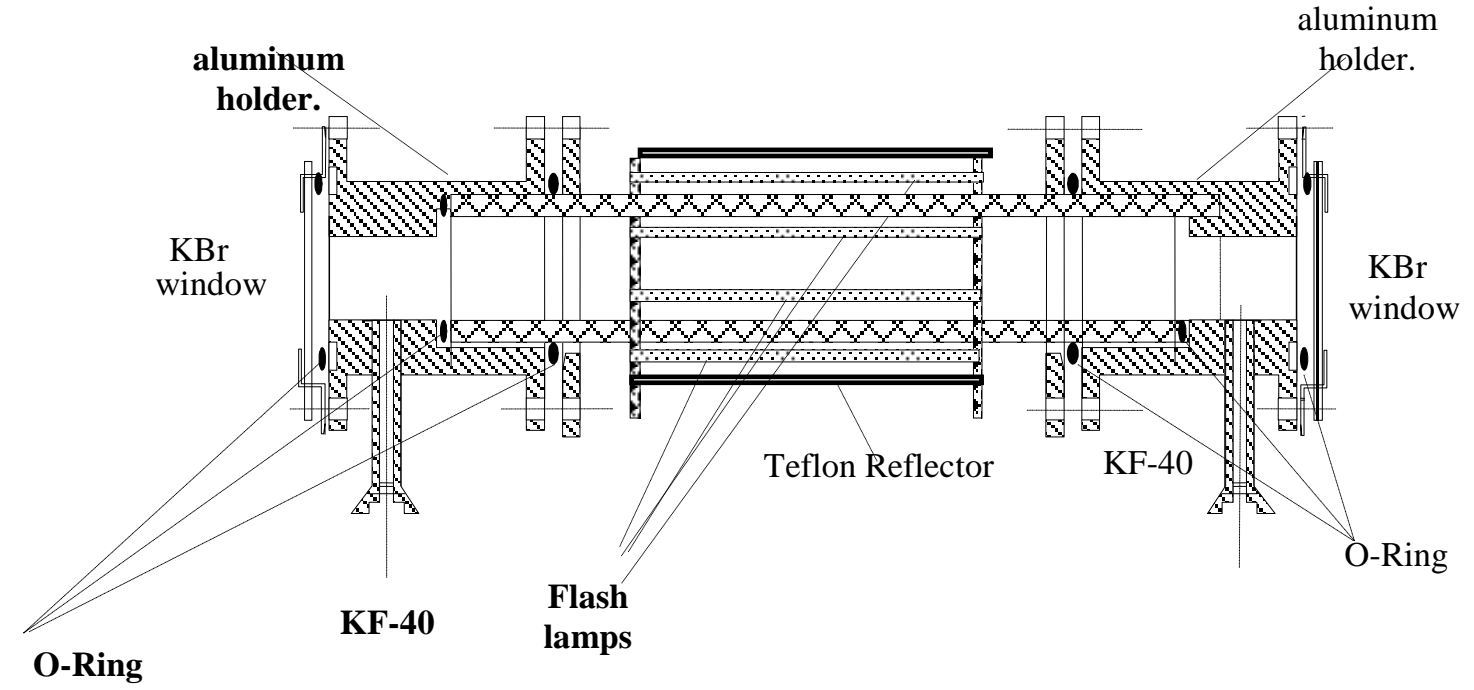

Fig. (3) Laser cavity aluminum window holders designed and constructed to fit and to seal the two ends of the quartz tube. aluminum holder. ndow

(radius of curvature) gold coated

A vacuum hood was used to mirror and flat sapphire window of evacuate and pull the gases if there are $63 \%$ reflectivity. Figs.(4), (5) shows any leaks. The active system with the resonator fixed inside the hood pictures of the chemical laser system. chamber which is consisted of $3 \mathrm{~m}$

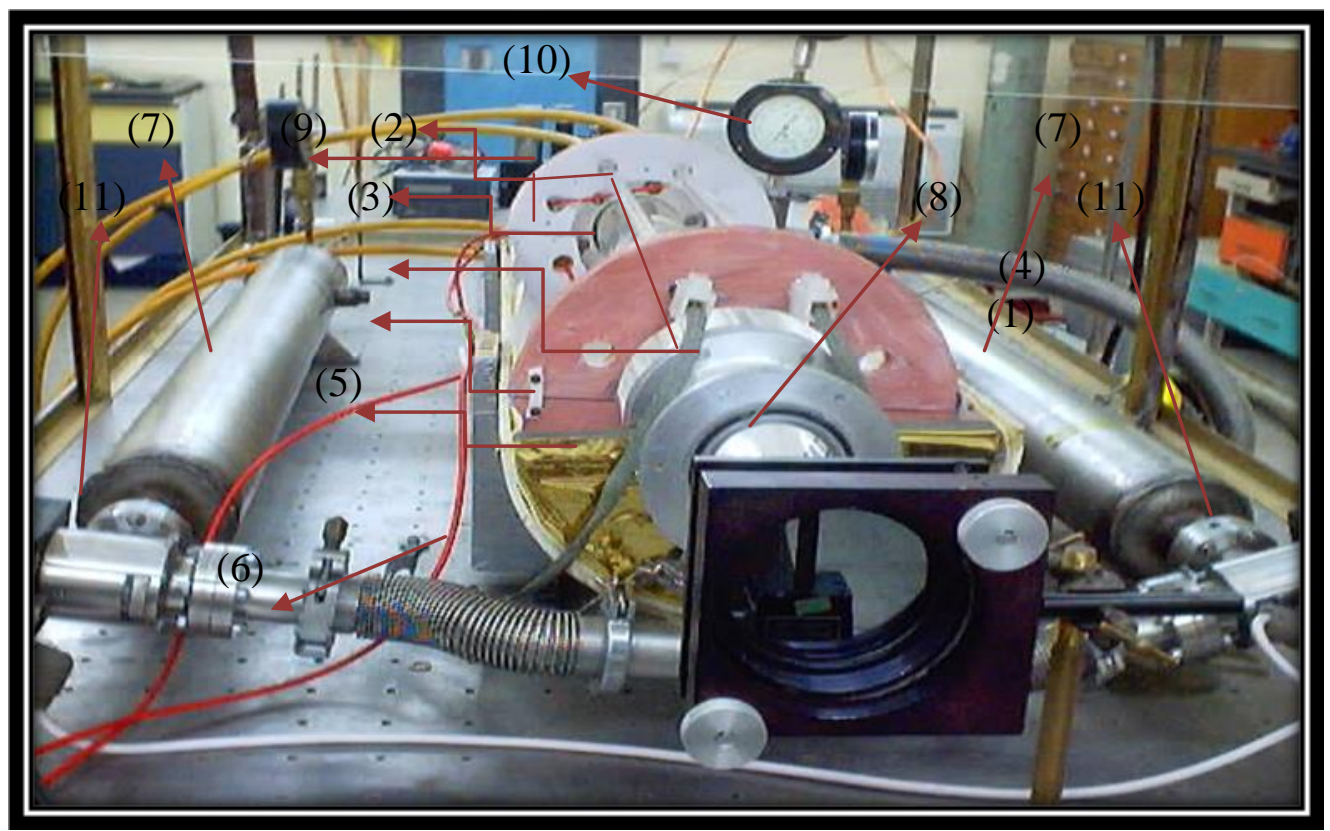

Fig(4)The system where the trigger and control circuits are shown.

$\underline{1-}$ Teflon Reflectr $\underline{2}$ - Flash lamps $\underline{3}$ - Laser cavity(quartz tube)

4- $\mathrm{KBr}$ window 5-aluminum holder. $\underline{6}$ - tube coneaction

$\underline{7}$ - gases mixture $\underline{8}$ - gold coated mirror $\underline{9}$ - flat sapphire window

10- gas gage 11-Pneumatic valves 


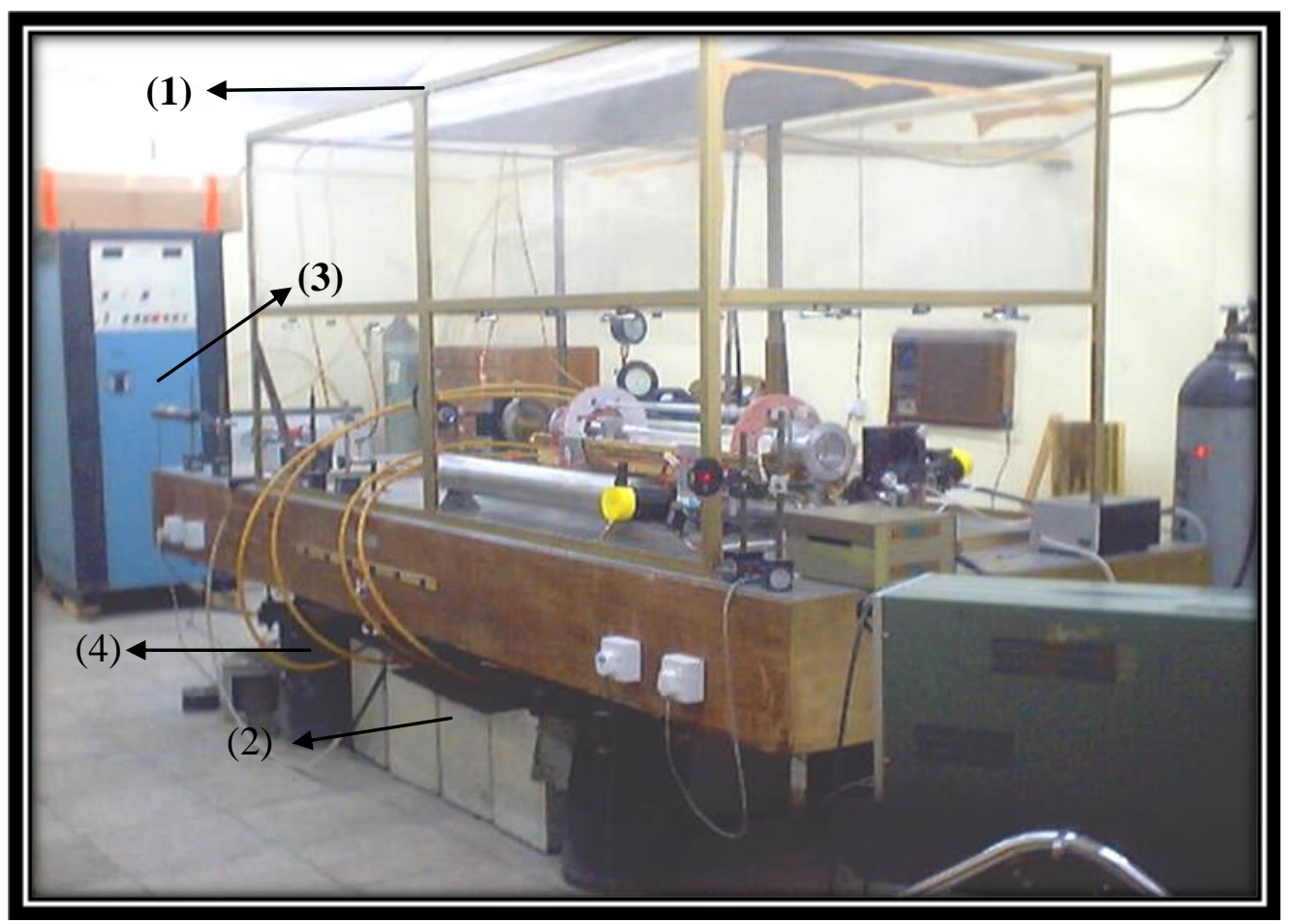

Fig(5)The whole system fixed in a chamber where a ventilation hood is assembled at the top of the chamber.

1-Ventilation hood 2- capacitors 3- power supply 4- rotary pump

\section{Results and Discussion:}

The energy of the laser pulse was recorded using a calorimeter type (ED500 from Genetic Corporation). The maximum output energy was $200 \mathrm{~mJ}$ at input energy of $(6400 \mathrm{~J})$. The laser pulse shape is shown in Fig.( 6). Many parameters are varied in order to get the optimum conditions for the laser pulse. The result of the laser pulse as a function of the total pressure for the mixed gases, satisfying the optimum ratio of the active gases 1:7:14 $\left(\mathrm{Cl}_{2}\right.$, $\mathrm{H}_{2}, \mathrm{He}$ ) are shown in figure (7). It is obvious that the laser energy depends dramatically on the total pressure. The laser energy is increased from $25 \mathrm{~mJ}$ at $50 \mathrm{mbar}$ of total pressure to $200 \mathrm{~mJ}$ when the pressure is increased to $125 \mathrm{mbar}$, then it is reduced to $150 \mathrm{~mJ}$ slowly when the total pressure is increased to $175 \mathrm{mbar}$.A rapid reduction in the laser pulse energy occurred when the total pressure is increased to $225 \mathrm{mbar}$. Our explanations can be divided into two main parts .

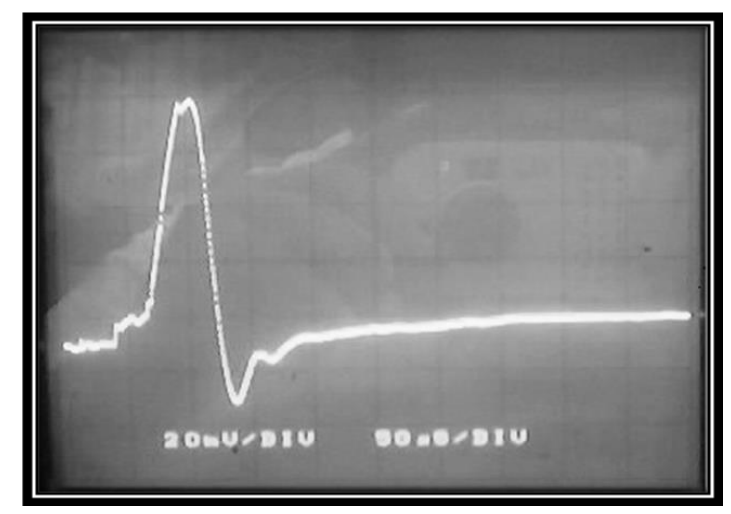

Fig. (6) The recorded laser pulse energy

1. In the first part, the energy pulse increases up to $200 \mathrm{~mJ}$ when the pressure reaches $125 \mathrm{mbar}$ and consequently, depends on the amount of the active product of molecules at certain electrical energy delivered to the active volume.

2. The second part of the curve can be explained as follow Since the electrical energy is fixed at $6400 \mathrm{~J}$, while the 
amount of active molecules increases due to the increase in the total pressure, the electrical energy was not enough to dissociate the excess molecules. On the other hand, the excess undissociated molecules will greatly affect the laser pulse energy. This is due to the collisions between the excited active molecules and the undissociated ones. From the explanation above, we expect that when the electrical energy increases to more than $6400 \mathrm{~J}$, the optimum laser pulse energy will shift towards the higher total pressure.

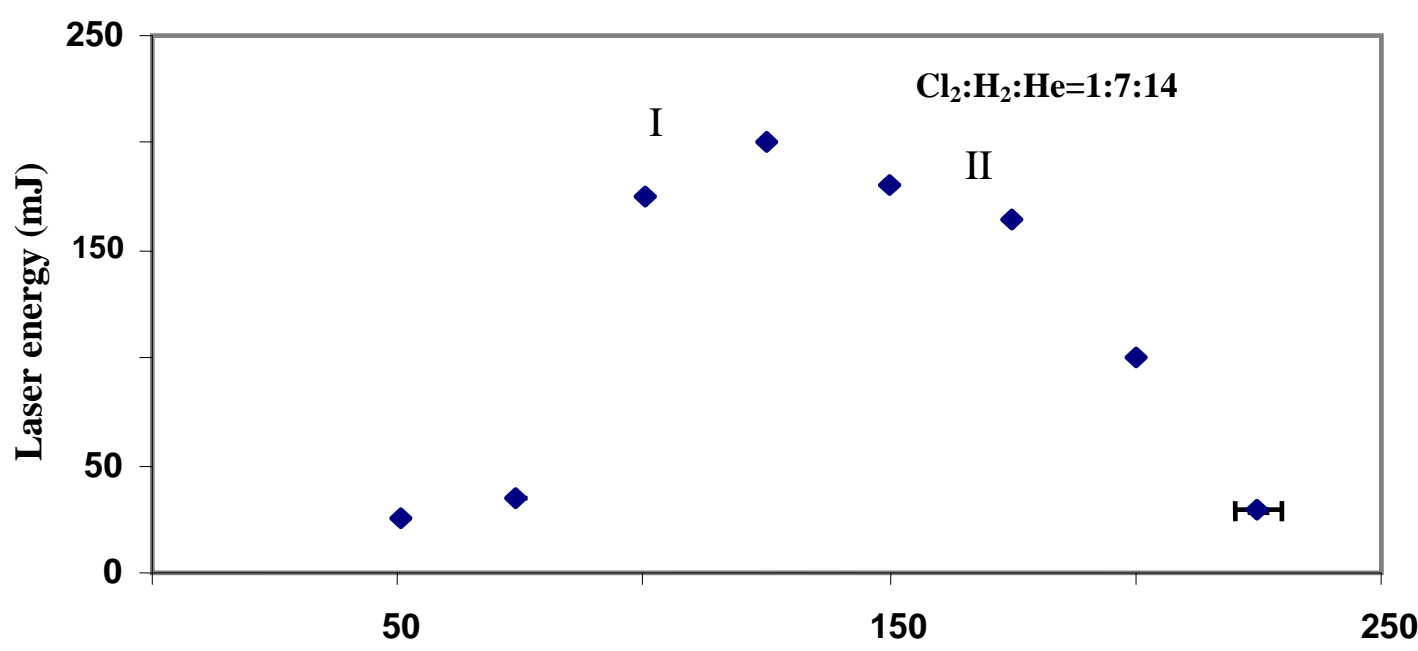

Total Pressure (mbar)

Fig.(7) The output pulse energy as a function of the total gases pressure

\section{Conclusions:}

Throughout the experimental research, we conclude the following points.

1. When the chlorine to Hydrogen ratio increased to more than $1: 3$, a violet glow inside the active medium was appeared after activating the system. This glowing lasts for 3 seconds. This kind of glowing is due to the reaction between $\mathrm{Cl}_{2}$ molecules in the excited state and the helium atoms which produced a dimmer in metastable state of long life time $(\mathrm{HeCl})$.

2. The reaction of $\mathrm{HCl}$ products, generates a shock wave due to the fast reaction of the active gases after the flash initiation. This shock sonic wave could causes an explosion was in the laser cavity.
3. The appearance of the shock wave severely affects the laser pulse. Accordingly, it is essential to reduce the shock wave to minimum value. This can be achieved when the pulse of the flash lamp is shorter than the time period of the sonic wave traveling toward the center of the tube.

\section{Reference:}

1- Vorob'er, A. P. and Iskhakov,V. A., 1998," Laser Diagnostics of Combustion Processes: From Chemical Dynamics to Technical Devices", IEEE J.Quant. Elec.,28 (7): 589-593.

2- Mirels, H. and Spencer. D. J. 1971,"Laser-induced fluorescence study of the hydrogen atom formation dynamics in the $248 \mathrm{~nm}$ gas phase photodisscoted of vibrational state selected water 
$\left(\mathrm{H}_{2} \mathrm{O}\right)$ "IEEE J. Quant. Elec. , 7 (11):513-522.

3- Rizhsky Pereulok, 1983, "Weaponry in space : The Dilemma of Security ", Mir Publishers, 2 Prevy, 1-110, GSP, Moscow, USSR 129820 .

4- .Gross R.W and J.F.Botl(ed) , 1976, "HandBook of Chemical Laser" , John Wiley \& Son,3 ed,205.

5- Joseph J.T, 1975, "A theoretical and experimental investigation of mechanism of the hydrogenfluoride pulsed chemical laser". Ph.D Thesis, Michigan State University, Physics, Optics. Xerox University microfilm.

6- Almuth L., Hans, R. V, Jaip,M and Rajesh, K,2007," Lyman- $\alpha$ photodissociation of $\mathrm{CH} 3 \mathrm{CFCl} 2$ (HCFC-141b): Quantum yield and translational energy of hydrogen atoms",J. Chem. Sci., 119( 4):277282.

7- Brown, R. A., Laurent ,T., Maity,D. K.and Vatsa. R. K., 2005," The reaction $\mathrm{ND}\left(\mathrm{a} 1 \_\right)+\mathrm{H} 2$ : laser spectroscopic measurement of room-temperature rate constant and $\mathrm{H} / \mathrm{D}$ atom product branching ratio", Res. Chem. Intermed., 31(1-3):193-203.

8- Gordon E.B., Matyushenko V.I., and Sizov V. D., 1997, " Laser
Diagnostics of Combustion

Processes: From Chemical Dynamics to Technical Devices", Applied energy: Russian Journal of Fuel, Power and Heat Systems , 35 ( 3): 81-90.

9- Melchior A., Knupfer P., Ilana B., and Salman R., Laurent T., Hans R. Volpp.P, and Wolfrum J., 1996," Photodissociation of $\mathrm{CHF} 2 \mathrm{Cl}$ at $193 \mathrm{~nm}: \mathrm{H} / \mathrm{Cl}$ and $\mathrm{Cl}(2 \mathrm{P} 1 / 2) / \mathrm{Cl}(2 \mathrm{P} 3 / 2) \quad$ Branching Ratios",J. Phys. Chem. 10(10): 13375-13380.

10-Gorski A., Yurkiv V., Bessler W.G.and Volpp H.-R., 2011, "Combined theoretical and experimental studies of $\mathrm{H}_{2}$ and $\mathrm{Co}$ oxidation over the YSZ surface " ,ECS Trans.,12(5):512-522.

11- Bravy B.G., Vasiliev G.K., Kiriganov V.I., Makarov E.F. and Chernysher Y.U.A, 2007, BRAS Physics/ supplement physics of vibrations, 60(2) :117-121.

12- Hanf .A., Volpp. H.-R., Sharma P., Mittal J. P.and Vatsa R. K., 2010, "Ultraviolet photochemistry of buta-1,3- and buta-1,2-dienes: Laser spectroscopic absolute hydrogen atom quantum yield and translational energy distribution measurements",,J. Chem. Phys. 133(3): 243-248 . 


\section{تصميم وبناء ليزر غازي يضخ ضوئياً}

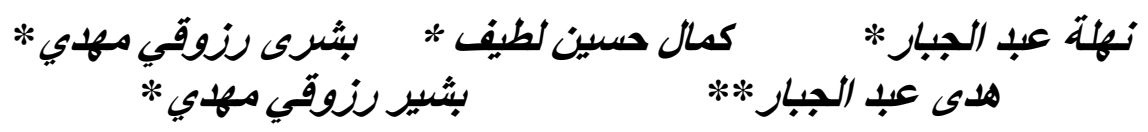

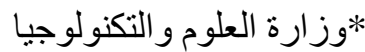

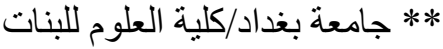

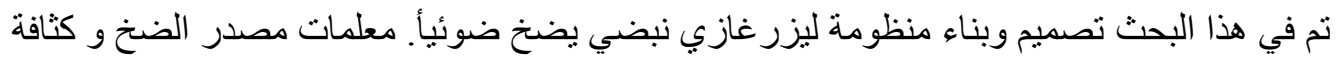

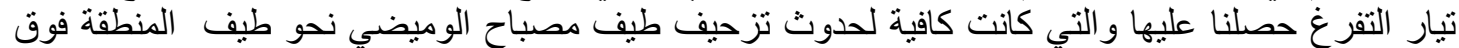

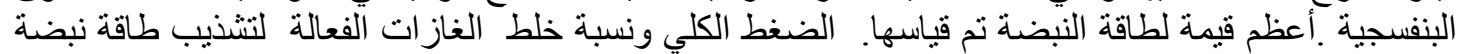

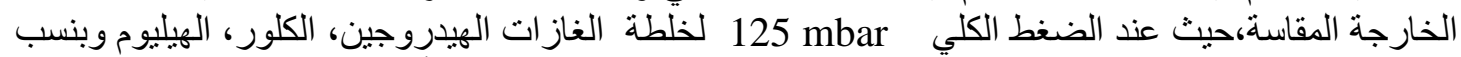
خلط( 1:7:14) وكانت نبضة لبزر بطاقة بحدود 200 mJ بطاقة ضخ لأربعة مصابيح وميضي حوالي لئيل

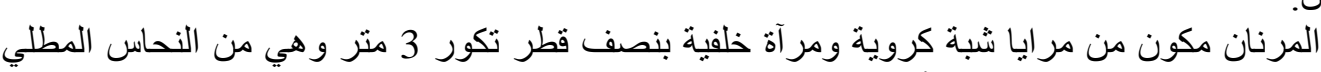

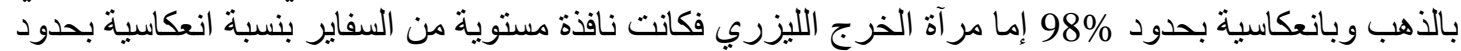

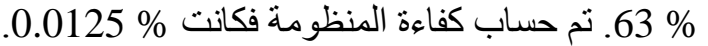

